\title{
SOME ASYMPTOTIC METHODS IN COMBINATORICS
}

\section{J. M. PLOTKIN and JOHN ROSENTHAL}

(Received 3 August 1978; revised 26 April 1979)

Communicated by W. D. Wallis

\begin{abstract}
Let $\left\langle f_{n}\right\rangle_{n} \geqslant 0$ be nonnegative real numbers with generating function $f(x)=\Sigma f_{n} x^{n}$. Assume $f(x)$ has the following properties: it has a finite nonzero radius of convergence $x_{0}$ with its only singularity on the circle of convergence at $x=x_{0}$ and $f\left(x_{0}\right)$ converges to $y_{0} ; y=f(x)$ satisfies an analytic identity $F(x, y)=0$ near $\left(x_{0}, y_{0}\right) ; F_{y(i)}\left(x_{0}, y_{0}\right)=0,0 \leqslant i<k$ and $F_{y(k)}\left(x_{0}, y_{0}\right) \neq 0$. There are constants $\gamma$, a positive rational, and $c$ such that $f_{n} \sim c x_{0}^{-n} n^{-(1+\gamma)}$. Furthermore, we show (i) in all cases how to determine $\gamma$ and $c$ from $f(x)$ and (ii) in certain cases how to determine them from $F(x, y)$.
\end{abstract}

1980 Mathematics subject classification (Amer. Math. Soc.): primary 05 A 15.

\section{$\mathbf{0}$}

Bender (1974) in his expository article and Harary, Robinson and Schwenk (1975) present similar methods of determining the asymptotic behavior of a sequence $\left\langle f_{n}\right\rangle_{n} \geqslant 0$ of nonnegative real numbers satisfying the following conditions.

Let $f(x)=\sum_{n \geqslant 0} f_{n} x^{n}$ be the generating function for $\left\langle f_{n}\right\rangle_{n} \geqslant 0$. Assume $f(x)$ has a finite nonzero radius of convergence $x_{0}$ with its only singularity on the circle of convergence at $x=x_{0}$ and furthermore that $f\left(x_{0}\right)$ converges to $y_{0}$. Assume also that there is a function $F(x, y)$ analytic near $\left(x_{0}, y_{0}\right)$ such that

(i) $F(x, f(x)) \equiv 0$ near $x_{0}$,

(ii) $F\left(x_{0}, y_{0}\right)=0, F_{y}\left(x_{0}, y_{0}\right)=0, F_{y y}\left(x_{0}, y_{0}\right) \neq 0$.

(Often as in Harary, Robinson and Schwenk (1975), one can use $F$ to show the only singularity of $f$ on its circle of convergence is at $x=x_{0}$.)

Bender (1974) shows that if $F_{x}\left(x_{0}, y_{0}\right) \neq 0$ then

$$
f_{n} \sim\left(x_{0} F_{x} / 2 \pi F_{y y}\right)^{1 / 2} n^{-3 / 2} x_{0}^{-n},
$$

where the partial derivatives are evaluated at $\left(x_{0}, y_{0}\right)$. Harary, Robinson and

The research for this paper was done while the second author was visiting Michigan State University and the writing while the second author was visiting the University of Sydney. 
Schwenk (1975) show that if

$$
\lim _{x \rightarrow x_{0}^{-}} f^{\prime}(x)\left(f\left(x_{0}\right)-j(x)\right) \neq 0
$$

then

$$
f_{n} \sim\left(b_{1} / 2\right)\left(x_{0} / \pi\right)^{1 / 2} n^{-3 / 2} x_{0}^{-n},
$$

where $b_{1}^{2} / 2$ is the value of the above limit. They also discuss a case where

$$
f_{n} \sim c \cdot n^{-5 / 2} x_{0}^{-n}
$$

for some $c$ which they indicate how to compute. Both results are generalizations of methods of Pólya (1937).

Recently Plotkin and Rosenthal found naturally occurring examples where instead of (ii) above holding we have for $k=4$

(ii) $F_{y(j)}\left(x_{0}, y_{0}\right)=0 \quad(0 \leqslant j \leqslant k-1)$,

$$
F_{y(k)}\left(x_{0}, y_{0}\right) \neq 0
$$

where $F_{y(j)}$ is $\partial^{j} F / \partial y^{j}$. These raise the question: Do results similar to those in Bender (1974) and Harary, Robinson, and Schwenk (1975) hold for $k>2$ ?

In Section 2 we derive results similar to those in Bender (1974) in that we read off the asymptotic behavior of $\left\langle f_{n}\right\rangle_{n} \geqslant 0$ from information on $F$ for certain cases in which $k>2$. In Section 3 we obtain results similar to those in Harary, Robinson and Schwenk (1975). But here we are able to read off the asymptotic behavior of $\left\langle f_{n}\right\rangle_{n \geqslant 0}$ from the information on $f$ for all cases in which $k>2$. Section 1 contains the necessary preliminaries for what follows.

Let $\left\langle f_{n}\right\rangle_{n \geqslant 0}$ be nonnegative real numbers and let $f(x)=\sum_{n \geqslant 0} f_{n} x^{n}$. Assume $f(x)$ has a finite nonzero radius of convergence $x_{0}$ with its only singularity on the circle of convergence at $x=x_{0}$ and that $f\left(x_{0}\right)$ converges to $y_{0}$. Assume there is a function $F(x, y)$ analytic near $\left(x_{0}, y_{0}\right)$ such that

(i) $F(x, f(x)) \equiv 0$ near $x_{0}$

(ii) $F_{y(j)}\left(x_{0}, y_{0}\right)=0 \quad(0 \leqslant j \leqslant k-1)$, $F_{y(k)}\left(x_{0}, y_{0}\right) \neq 0$.

We generalize the proof of Bender (1974), p. 505. By the Weierstrass preparation theorem (see, for example, Hörmander (1966), p. 144) near $\left(x_{0}, y_{0}\right)$

$$
F(x, y)=U(x, y) P(x, y)
$$

where

(a) $U(x, y)$ is analytic, nonvanishing near $\left(x_{0}, y_{0}\right)$; and

(b) $P(x, y)$ is a function which is a monic polynomial of degree $k$ in $\left(y-y_{0}\right)$ such 
that for each $j<k$ the coefficient $p_{j}(x)$ of $\left(y-y_{0}\right)^{j}$ is a function vanishing at and analytic near $x_{0} . P(x, y)$ is called a Weierstrass polynomial.

From the factorization of $F$ and (i) and (a)

$$
P(x, f(x)) \equiv 0 \quad \text { near } x_{0} .
$$

Hence $f(x)-y_{0}$ can be expressed as a fractional power series (see, for example, Walker (1950), p. 98)

$$
\sum_{i=1}^{\infty} f_{i}(x)\left(1-\frac{x}{x_{0}}\right)^{r_{i}} \quad\left(\text { or } \sum_{i=1}^{m} f_{i}(x)\left(1-\frac{x}{x_{0}}\right)^{r_{i}}\right)
$$

where

(i) $r_{i}$ is an increasing sequence of rational numbers and

(ii) $f_{i}(x)$ is analytic nonvanishing near $x=x_{0}$.

(The branch of $\left(1-\left(x / x_{0}\right)\right)^{r_{i}}$ we are using may be chosen to be the one for which $\left(1-\left(x / x_{0}\right)\right)^{r_{t}}$ is real for $x$ real, near to and less than $x_{0}$.)

Thus $f(x)$ can be expressed as

$$
\sum_{i=1}^{\infty} a_{i}\left(1-\frac{x}{x_{0}}\right)^{s_{i}} \quad\left(\text { or } \sum_{i=1}^{m} a_{i}\left(1-\frac{x}{x_{0}}\right)^{s_{i}}\right)
$$

where

(i) $s_{i}$ is an increasing sequence of rational numbers and

(ii) $a_{i}$ are nonzero.

By a special case of a theorem of Darboux (see Bender (1974), Theorem 4) we have

$$
f_{n} \sim\left(a_{p} / \Gamma\left(-s_{p}\right)\right) x_{0}^{-n} n^{-\left(1+s_{p}\right)},
$$

where

(i) $s_{p}$ is the least noninteger amongst the $s_{i}$ 's; and

(ii) $\Gamma$ is the classical gamma function.

(We know some $r_{i}$, and hence some $s_{i}$, is nonintegral as $f$ has a singularity at $x_{0}$. Furthermore, as $f\left(x_{0}\right)$ converges all $r_{i}$ are positive.)

Alternately we have

$$
f_{n} \sim\left(f_{q}\left(x_{0}\right) / \Gamma\left(-r_{q}\right)\right) x_{0}^{-n} n^{-\left(1+r_{q}\right)}
$$

where $r_{q}$ is the least noninteger amongst the $r_{i}$ 's.

To generalize the results in Bender (1974) and Harary, Robinson and Schwenk (1975) it suffices to indicate how to find $s_{p}$ and how to compute $a_{p}$. This will be done by examining more carefully how the fractional power series is obtained using the method of Newton polygons. 
Let $n_{j}$ be the order of the zero at $x=x_{0}$ of $p_{j}(x)$, the coefficient of $\left(y-y_{0}\right)^{j}$ in the Weierstrass polynomial $P(x, y)$. Say $p_{j}(x)=\sum_{i \geqslant n_{j}} p_{j i}\left(x-x_{0}\right)^{i}$ where $p_{j n_{j}} \neq 0$ (if $p_{j}(x) \equiv 0$, we let $n_{j}=+\infty$ ). Let $n_{k}=0$.

Since

$$
0=P(x, f(x))=\left(f(x)-y_{0}\right)^{k}+\sum_{j=0}^{k-1} p_{j}(x)\left(f(x)-y_{0}\right)^{j},
$$

in order that the lowest degree term in $\left(x-x_{0}\right)$ cancels when we substitute $\sum a_{i}\left(1-\left(x / x_{0}\right)\right)^{s_{i}}$ for $f(x)-y_{0}$ we must have that two of the $\left\{n_{i}+i s_{1} \mid 0 \leqslant i \leqslant k\right\}$ are equal and all others are at least as large. Thus $s_{1}=\left(n_{i_{1}}-n_{i_{2}}\right) /\left(i_{2}-i_{1}\right)$ for some $0 \leqslant i_{1}<i_{2} \leqslant k$. So $s_{1}$ is expressible as a rational number with denominator $\leqslant k$.

We have shown:

Lemma 2.1. If $P(x, f(x)) \equiv 0$ near $x_{0}$ where $P(x, y)$ is a function which is a monic polynomial of degree $k$ in $\left(y-y_{0}\right)$, then the denominator of the lowest degree term in a fractional power series expansion of $f(x)$ about $x_{0}$ can be taken to be at most $k$.

In fact from the above representation of $s_{1}$ we see that the denominator is $k$ if and only if $n_{0}=k s_{1}$ and $n_{i}+i s_{1} \geqslant k s_{1}, 0<i<k$, if and only if $n_{i} \geqslant(1-i / k) n_{0}$, $0<i<k$. The $n_{i}$ 's are determined by $P(x, y)$ as follows:

LemMa 2.2. $n_{i}$ is the least $p$ such that

$$
P_{x^{(p)} y^{(i)}}\left(x_{0}, y_{0}\right) \neq 0 .
$$

Corollary 2.3. $s_{1}=n_{0} / k$ if and only if

$$
P_{x(p)}\left(x_{0}, y_{0}\right) \begin{cases}=0, & p<n_{0} \\ \neq 0, & p=n_{0},\end{cases}
$$

and

(ii) For $0<i<k$ and $p<(1-i / k) n_{0}$

$$
P_{x^{(p) y(1)}}\left(x_{0}, y_{0}\right)=0 \text {. }
$$

Even though $P(x, y)$ cannot be effectively obtained from $F(x, y), F$ yields significant information about $P$.

Proposition 2.4. $s_{1}=n_{0} / k$ if and only if

$$
F_{x(p)}\left(x_{0}, y_{0}\right) \begin{cases}=0, & p<n_{0} \\ \neq 0, & p=n_{0}\end{cases}
$$


and

(ii) For $0<i<k$ and $p<(1-i / k) n_{0}$

$$
F_{x(p) y(i)}\left(x_{0}, y_{0}\right)=0 \text {. }
$$

Proof. Assume $s_{1}=n_{0} / k$. By Leibniz's rule for derivatives of products we can express $F_{x^{(p)} y^{(i)}}\left(x_{0}, y_{0}\right)$ in terms of $P_{x^{(q)} y^{(j)}}\left(x_{0}, y_{0}\right)$ for $q \leqslant p, j \leqslant i$ and $U$ and its derivatives. Inspection then shows (i) and (ii) of 2.4 hold as (i) and (ii) of 2.3 hold. (We use the fact that $(1-i / k) n_{0}$ is decreasing in $i$ )

Conversely (i) and (ii) of 2.4 imply (i) and (ii) of 2.3 . If (i) of 2.3 fails, then we have

$$
P_{x(p)}\left(x_{0}, y_{0}\right) \begin{cases}=0, & p<n_{0}^{\prime}, \\ \neq 0, & p=n_{0}^{\prime},\end{cases}
$$

where $n_{0}^{\prime} \neq n_{0}$; and hence by what we have just proved the analogous statement will be true for $F_{x(p)}\left(x_{0}, y_{0}\right)$. If (i) of 2.3 holds but (ii) of 2.3 fails, let $i_{0}$ be the least integer for which it fails and let $r$ be the least integer for which

$$
P_{x(r) y(t(0))}\left(x_{0}, y_{0}\right) \neq 0 \text {. }
$$

Inspection of $F_{x^{(r)} y_{(i(0))}}\left(x_{0}, y_{0}\right)$ expressed in terms of

$$
P_{\left.x^{(a)}\right)^{(j)}}\left(x_{0}, y_{0}\right), q \leqslant r, j \leqslant i_{0} \text { and } U
$$

and its derivatives shows it is also nonzero.

In the case where $s_{1}=n_{0} / k$ is not an integer it remains to compute $a_{1}$. If we substitute $f(x)$ for $y$ in the power series expansion of $F(x, y)$ about $\left(x_{0}, y_{0}\right)$, by 2.4 the lowest power of $\left(x-x_{0}\right)$ obtained is $\left(x-x_{0}\right)^{n_{0}}$. Since $F(x, f(x)) \equiv 0$ near $x_{0}$, we have by computing the coefficient of $\left(x-x_{0}\right)^{n}$ that

$$
\begin{aligned}
\text { (*) } F_{x^{n_{0}}} \cdot \frac{1}{n_{0} !}+ & \sum\left\{F_{x^{\left((1-j / k) n_{0} y(j)\right)}} \frac{1}{\left((1-j / k) n_{0}\right) ! j !}\left(\frac{a_{1}}{\left(-x_{0}\right)^{s_{1}}}\right) j \mid\right. \\
& +F_{y(k)} \frac{1}{k !}\left(\frac{a_{1}}{\left(-x_{0}\right)^{s_{1}}}\right)^{k}=0,
\end{aligned}
$$

where the derivatives are evaluated at $\left(x_{0}, y_{0}\right)$.

It remains to solve this equation for $a_{1}$. In general we cannot determine which solution is the desired one. However, if there is only one solution for which $a_{1} / \Gamma\left(-s_{1}\right)$ is positive real, we may conclude

$$
f_{n} \sim\left(a_{1} / \Gamma\left(-s_{1}\right)\right) x_{0}^{-n} n^{-\left(1+s_{1}\right)}
$$


For example:

THEOREM 2.5. Let $\left\langle f_{n}\right\rangle_{n} \geqslant 0$ be nonnegative real numbers such that

(i) $f(x)=\sum_{n} \geqslant 0 f_{n} x^{n}$ has a finite nonzero radius of convergence $x_{0}$ with its only singularity on the circle of convergence at $x=x_{0}$.

(ii) $f\left(x_{0}\right)$ converges to $y_{0}$.

(iii) There is a function $F(x, y)$ analytic near $\left(x_{0}, y_{0}\right)$ and relatively prime integers $n_{0}$ and $k$ such that

(a) $F(x, f(x)) \equiv 0$ near $x_{0}$,

(b) $F_{y(j)}\left(x_{0}, y_{0}\right) \begin{cases}=0, & 0 \leqslant j \leqslant k-1, \\ \neq 0, & j=k,\end{cases}$

(c) $F_{x^{(p)}}\left(x_{0}, y_{0}\right) \begin{cases}=0, & 0 \leqslant p<n_{0} \\ \neq 0, & p=n_{0}\end{cases}$

(d) $F_{x(p) y(j)}\left(x_{0}, y_{0}\right)=0, \quad p<(1-j / k) n_{0}$,

then

$$
f_{n} \sim\left(a_{1} / \Gamma\left(-n_{0} / k\right)\right) \cdot x_{0}^{-n} n^{-\left(1+n_{0} / k\right)} .
$$

where $a_{1}$ is the solution of

$$
F_{x^{(n(0))}} \frac{1}{n_{0} !}+F_{y(k)} \frac{1}{k !\left(-x_{0}\right)^{n_{0}}}=0
$$

such that $a_{1} / \Gamma\left(-n_{0} / k\right)$ is positive real.

PROOF. As $k$ and $n_{0}$ are relatively prime $(1-j / k) n_{0}$ is not an integer for $1 \leqslant j \leqslant k-1$. Thus $\left(^{*}\right)$ reduces to the above equation.

As in Section 2 we know $s_{1}$ is a positive rational number expressible with denominator $\leqslant k$. Say $s_{1}=m / n$ in reduced form. $s_{1}$ is characterized by

LEMMA 3.1.

$$
\lim _{x \rightarrow x_{0}-} \frac{d^{p}}{d x^{p}}\left(\left(f(x)-y_{0}\right)^{q}\right)= \begin{cases}0, & 0<p / q<m / n, \\ \infty, & p / q>m / n, \\ \text { finite, nonzero, }, & p / q=m / n,\end{cases}
$$

where $p, q$ are positive integers. In particular if $p=m$ and $q=n$ the limit is $a_{1}^{n} m ! /\left(-x_{0}\right)^{m}$. 
Proof. This may be proven by using the expansion of $f(x)-y_{0}$ as

$$
\sum a_{i}\left(1-\frac{x}{x_{0}}\right)^{s_{i}}
$$

to obtain a similar fractional power series expansion of

$$
\frac{d^{p}}{d x^{p}}\left(\left(f(x)-y_{0}\right)^{q}\right)
$$

To determine if $s_{1}$ is nonintegral and in that case to obtain the asymptotic behavior of $\left\langle f_{n}\right\rangle_{n} \geqslant 0$ one may proceed as follows:

Step 1. Compute $F_{y^{(k)}}\left(x_{0}, y_{0}\right)$ until we find the least $k$ for which it is nonzero. This gives the maximum possible denominator for $s_{1}$.

Step 2. Compute

$$
\lim _{x \rightarrow x_{0}-} \frac{d^{l}}{d x^{l}}\left(f(x)-y_{0}\right)
$$

for each nonnegative integer $l$ until either (i) the value is finite nonzero or (ii) the value is infinite. In case (i) we have $s_{1}$ is integral. We return to this case later. In case (ii) $s_{1}$ lies strictly between $l-1$ and $l$.

Step 3 . There are only finitely many rational numbers between $l-1$ and $l$ with denominator $\leqslant k$. Find out for which one, $m / n$, of these

$$
\lim _{x \rightarrow x_{0}-} \frac{d^{m}}{d x^{m}}\left(\left(f(x)-y_{0}\right)^{n}\right)
$$

is finite nonzero. This is $s_{1}, a_{1}$ is a solution of

$$
\frac{a_{1}^{n} m !}{\left(-x_{0}\right)^{m}}=\lim _{x \rightarrow x_{0}-} \frac{d^{m}}{d x^{m}}\left(\left(f(x)-y_{0}\right)^{n}\right)
$$

As in Section 2, since $f_{n} \sim\left(a_{1} / \Gamma\left(-s_{1}\right)\right) x_{0}^{-n} x^{-\left(1+s_{1}\right)}, a_{1}$ must be the unique solution such that $a_{1} / \Gamma\left(-s_{1}\right)$ is positive real.

Observe in Step 2, if $s_{1}$ turns out to be an integer $l$, then $a_{1}$ is determined by

$$
\frac{a_{1} l !}{\left(-x_{0}\right)^{l}}=\lim _{x \rightarrow x_{0}-} \frac{d^{l}}{d x^{l}}\left(\left(f(x)-y_{0}\right)\right) \text {. }
$$

In the case where $s_{1}$ is an integer we call the above three steps Stage 1.

Now let us assume for the sake of induction that $s_{1}, \ldots, s_{t}$ are integers and that we have found $a_{1}, \ldots, a_{t}$. We show how to determine whether $s_{t+1}$ is an integer and how to find $a_{t+1}$. 
Let

$$
L(x)=\sum_{i=1}^{t} a_{i}\left(1-\frac{x}{x_{0}}\right)^{s_{i}}
$$

and let $g(x)=f(x)-L(x)$.

LEMMA 3.2. $s_{t+1}$ can be written with a denominator $\leqslant k$.

Proof. Let $P(x, y)$ be the Weierstrass polynomial of degree $k$ in $\left(y-y_{0}\right)$ obtained in Section 1. Since $P(x, f(x)) \equiv 0$ near $x_{0}$ we have $P(x, g(x)+L(x)) \equiv 0$ near $x_{0}$. Since $s_{1}, \ldots, s_{t}$ are integers, by the binomial expansion of $(g(x)+L(x))^{j}$ we can obtain a similar function $Q(x, y)$ which is a monic polynomial of degree $k$ in $\left(y-y_{0}\right)$ and such that $Q(x, g(x)) \equiv 0$ near $x_{0}$. We are now done by 2.1 .

\section{LEMMA 3.3.}

$$
\lim _{x \rightarrow x_{0}-} \frac{d^{p}}{d x^{p}}\left(\left(g(x)-y_{0}\right)^{q}\right)= \begin{cases}0, & 0<p / q<m / n \\ \infty, & p / q>m / n, \\ \text { finite, nonzero, }, & p / q=m / n,\end{cases}
$$

where $p, q$ are integers. In particular if $p=m, q=n$ the limit is

$$
\frac{\left(a_{t+1}\right)^{n} m !}{\left(-x_{0}\right)^{m}}
$$

Proof. This is proven just as in 3.1.

\section{Stage $t+1$}

Step 1. Compute

$$
\lim _{x \rightarrow x_{0}} \frac{d^{l}}{d x^{l}}\left(g(x)-y_{0}\right)
$$

for each nonnegative integer $l>s_{t}$ until either (i) the value is finite, nonzero or (ii) the value is infinite. In case (i) $s_{t+1}$ is integral and we can find $a_{t+1}$ from

$$
\frac{a_{t+1} l !}{\left(-x_{0}\right)^{l}}=\lim _{x \rightarrow x_{0}-} \frac{d^{l}}{d x^{l}}\left(g(x)-y_{0}\right) .
$$

In case (ii) $s_{t+1}$ lies strictly between $l-1$ and $l$.

Step 2. This is just like Step 3 of Stage 1 except we use $g(x)$ throughout instead of $f(x)$ and we are determining $s_{t+1}$ and $a_{t+1}$ instead of $s_{1}$ and $a_{1}$. 


\section{References}

E. A. Bender (1974), 'Asymptotic methods in enumeration', Siam Review 16, 485-515.

F. Harary, R. W. Robinson and A. J. Schwenk (1975), 'Twenty step algorithm for determining the asymptotic number of trees of various species', J. Austral. Math. Soc. Ser. A 20, 483-503.

L. Hörmander (1966), An introduction to complex analysis in several variables (Van Nostrand, Princeton).

J. M. Plotkin and J. Rosenthal (1979), 'On the expected number of branches in analytic tableaux analyses in propositional calculus' (preprint).

G. Pólya (1937), 'Kombinatorische Anzahlbestimmungen für Gruppen, Graphen und chemische Verbindungen', Acta Math. 68, 145-254.

R. J. Walker (1950), Algebraic curves (Princeton University Press, Princeton).

$\begin{array}{ll}\text { Michigan State University } & \text { Ithaca College } \\ \text { East Lansing 48824 } & \text { Ithaca } 14850 \\ \text { USA } & \text { USA }\end{array}$

